\title{
SPARSE NEAR-EQUIANGULAR TIGHT FRAMES WITH APPLICATIONS IN FULL DUPLEX WIRELESS COMMUNICATION
}

\author{
A. Thompson \\ Mathematical Institute \\ University of Oxford \\ Oxford, United Kingdom
}

\author{
R. Calderbank \\ Department of ECE \\ Duke University \\ Durham, North Carolina
}

\begin{abstract}
We construct extremely sparse, near-equiangular tight frames which share the same row space as certain incomplete Delsarte-Goethals frames. Frames combining these properties have application in full duplex communication in ad-hoc wireless networks. We highlight their computational advantage over similar constructions of sparse equiangular tight frames: namely that their associated matrix-vector products can be implemented as a fast transform.
\end{abstract}

Index Terms - Equiangular tight frames, sparse frames, Delsarte Goethals frames, fast transforms, full duplex communication.

\section{INTRODUCTION}

An equiangular tight frame (ETF) is an optimal packing of $N$ lines in $n$-dimensional Euclidean space. More precisely, we seek an $n \times N$ matrix $A$ with unit norm columns $\left\{a_{i}\right\}$ with minimum coherence $\mu(A)$, defined to be

$$
\mu(A):=\min _{i \neq j}\left|\left\langle a_{i}, a_{j}\right\rangle\right| .
$$

It is well-known that $\mu(A)$ satisfies the Welch bound [19]

$$
\mu(A) \geq \sqrt{\frac{N-n}{n(N-1)}},
$$

with equality attained if and only if it is a tight frame (its rows are orthogonal) and equiangular (all abso- lute values of inner products $\left|\left\langle a_{i}, a_{j}\right\rangle\right|$ between distinct columns are equal) [17].

ETFs are a natural way to combine the advantages of orthonormal bases with those of redundant representations, and they have numerous practical applications. In coding theory, they have been shown to provide error correcting codes which are maximally robust to erasures $[14,6]$. They also have applications in waveform design for wireless communications such as code-division multiple access (CDMA) [17], and compressive sensing [11].

While ETFs have proved notoriously challenging to construct [18], several infinite families are now known. The nontrivial infinite families can be grouped into three categories: designs based on conference matrices [17], harmonic ETFs [17], and designs based on balanced incomplete block designs (for example Steiner systems) [12, 15]. At the same time, a number of infinite families of near-equiangular tight frames consisting of unions of orthonormal bases have been constructed which nearly attain the Welch bound. Examples of such constructions include discrete chirp frames [5], Alltop Gabor frames [1] and Delsarte-Goethals frames [8]; the latter will be the inspiration for our construction of sparse near-equiangular tight frames in this paper. If it is required that $N>n^{2}$, it is known that ETFs cannot exist, and the aforementioned near-equiangular frames give optimal line packings [10].

There has been recent interest in sparse equiangular (or near-equiangular) tight frames. The exis- 
tence and construction of sparse fusion frames (collections of subspaces) was studied in [7], while the concept of optimally sparse frames was introduced in [9]. In addition, the infinite families of ETFs based on Steiner systems [12] are extremely sparse.

In this paper, we propose a construction of sparse near-equiangular tight frames, which we refer to as Sparse Kerdock Frames. We define certain incomplete real Delsarte-Goethals (DG) frames and show that applying an orthogonal transformation to the rows of the matrix results in an extremely sparse matrix. We obtain an infinite family of matrices of size $n \times n^{3 / 2}$ (where $n$ is an even power of 2), such that the fraction of nonzeros in each column is $1 / \sqrt{n}$. Since Sparse Kerdock Frames are obtained from DG frames by a left orthogonal transformation, it follows that they share the same Gram matrix, pairwise column inner products and coherence. They also share precisely the same row space and null space as the DG frames from which they are derived, projecting into the same low-dimensional subspace, and therefore one would expect them to perform analogously to DG frames in compressive sensing applications [8].

Our construction is motivated in part by the framework proposed by Guo and Zhang [13] for full duplex communication in ad-hoc wireless networks. In this framework, an individual node in the wireless network either transmits or it listens to transmissions from other nodes but it cannot do both at the same time. There might be as many nodes as there are 48 bit MAC addresses but it is assumed that only a small subset of nodes contribute to the superposition received at any given node in the network. Ideas from compressive sensing are then used to show that simultaneous communication is possible across the entire network.

Fundamental to [13] is the idea that it is better to manage interference through configuration rather than to eliminate or align it through extensive exchange of fine-grained Channel State Information. In fact Guo and Zhang [13] developed an information theoretic analysis of the capacity of full duplex communication, and demonstrated that the performance scales with the number of users better than that of ALOHA type protocols. We construct a deterministic matrix, where the rows correspond to time slots, the columns to different users, and where the pattern of zero and nonzero entries describes how nodes transmit and receive in concert to achieve full duplex communication without transmitter coordination. In this application it is assumed that only a small subset of nodes contribute to the superposition received at any given node in the network (see [3, 2, 4] for more details on implementation).

We also discuss two particular advantages that Sparse Kerdock Frames have over other known infinite families of sparse ETFs, namely Steiner ETFs [12].

1. Real-valued frames are often desirable in communications applications. Sparse Kerdock Frames are always real-valued, whereas Steiner ETFs with a comparable number of columns can never be real.

2. Compressive sensing reconstruction algorithms typically require the repeated computation of matrix-vector products (mat-vecs) associated with a frame. Mat-vecs involving Sparse Kerdock Frames can be implemented with $\mathcal{O}(N \log N)$ complexity by making use of the Walsh-Hadamard Transform, compared to $\mathcal{O}\left(N^{4 / 3}\right)$ for Steiner ETFs.

An outline of the paper is as follows. Section 2 describes the construction of Sparse Kerdock Frames. We first give background on DelsarteGoethals frames, before defining Sparse Kerdock Frames and describing their sparsity properties. Section 3 discusses the advantages of Sparse Kerdock Frames over other families of sparse ETFs.

\section{SPARSE KERDOCK FRAMES}

Delsarte-Goethals (DG) frames were constructed in [8] by exponentiating codewords of the secondorder Reed-Muller code. Indexing rows by $u \in \mathbb{Z}_{2}^{m}$ and columns by $\left(\begin{array}{ll}a & b\end{array}\right)$ where $a, b \in \mathbb{Z}_{2}^{m}$, define a $2^{m} \times 2^{2 m}$ matrix $J^{m}$ to have entries

$$
\left\{J^{m}\right\}_{u,(a, b)}:=\frac{1}{2^{m / 2}} i^{2 u a^{T}+u P_{b} u^{T}}
$$


where the exponent is calculated over $\mathbb{Z}_{4}$, and where $\left\{P_{b}\right\}$ is a Kerdock set of $2^{m}$ binary symmetric matrices of size $m \times m$ having the property that $P_{b_{1}}+P_{b_{2}}$ is full rank over $\mathbb{Z}_{2}$ for all distinct $b_{1}, b_{2} \in \mathbb{Z}_{2}^{m}[16]$. $J^{m}$ is a union of orthobases, namely

$$
J^{m}=\left(\begin{array}{llll}
D_{1} H_{2^{m}} & D_{2} H_{2^{m}} & \ldots & D_{2^{m}} H_{2^{m}}
\end{array}\right),
$$

where $D_{1}, D_{2}, \ldots$ are diagonal matrices and $H_{2}$ is the $2^{m} \times 2^{m}$ Hadamard matrix with entries

$$
\left\{H_{2^{m}}\right\}_{u, a}=\frac{1}{2^{m / 2}}(-1)^{u a^{T}} .
$$

$J^{m}$ is thus clearly a tight frame, but moreover it is near-equiangular with $\mu\left(J^{m}\right)=2^{-m / 2}$.

We now consider an incomplete DG frame defined as follows. We square the dimensions of the orthobases, indexing the rows by $u \in \mathbb{Z}_{2}^{2 m}$ and the columns of each orthobasis by $a \in \mathbb{Z}_{2}^{2 m}$. We consider a set $\left\{Q_{b}\right\}$ of $2^{m}$ binary symmetric matrices of size $2 m \times 2 m$ of the form

$$
Q_{b}:=\left(\begin{array}{cc}
0 & P_{b} \\
P_{b} & 0
\end{array}\right)
$$

where $\left\{P_{b}\right\}$ is a (complete) Kerdock set indexed by $b \in \mathbb{Z}_{2}^{m}$. The set $\left\{Q_{b}\right\}$ is an incomplete Kerdock set since the Kerdock property, namely that sums of distinct matrix elements are full rank over $\mathbb{Z}_{2}$, is inherited from $\left\{P_{b}\right\}$. Following (1), we obtain a $2^{2 m} \times 2^{3 m}$ matrix $K^{m}$ with entries given by

$$
K_{u,(a, b)}^{m}=\frac{1}{2^{m}} i^{2 u a^{T}+u Q_{b} u^{T}} .
$$

Writing $u=\left(\begin{array}{ll}u_{1} & u_{2}\end{array}\right)$ and $a=\left(\begin{array}{ll}a_{1} & a_{2}\end{array}\right)$, where $u_{1}, u_{2}, a_{1}, a_{2} \in \mathbb{Z}_{2}^{m}$, we have

$u Q_{b} u^{T}=\left(\begin{array}{ll}u_{1} & u_{2}\end{array}\right)\left(\begin{array}{cc}0 & P_{b} \\ P_{b} & 0\end{array}\right)\left(\begin{array}{l}u_{1} \\ u_{2}\end{array}\right)=2 u_{1} P_{b} u_{2}^{T}$,

where the last step follows from the symmetry of $P_{b}$. Since the quadratic form is even, the DG frame is real in this case, and we may rewrite (2) as

$$
K_{\left(u_{1}, u_{2}\right),\left(a_{1}, a_{2}, b\right)}^{m}=\frac{1}{2^{m}}(-1)^{u_{1}^{T} a_{1}+u_{2}^{T} a_{2}+u_{1}^{T} P_{b} u_{2}} .
$$

Just like $J^{m}, K^{m}$ is a near-equiangular tight frame, though with a reduced number of columns in relation to the number of rows.
We now define another matrix $S^{m}$, also of size $2^{2 m} \times 2^{3 m}$, which is obtained from $K^{m}$ by a simple orthogonal transformation: define

$$
S^{m}:=\left(H_{2^{m}} \otimes I_{2^{m}}\right) K^{m},
$$

where $I_{2^{m}}$ is the $2^{m} \times 2^{m}$ identity matrix and $\otimes$ denotes the Kronecker product.

It follows immediately that $S^{m}$ shares the same row space and nullspace as $K^{m}$. Moreover, $S^{m}$ and $K^{m}$ share the same Gram matrix, that is

$$
\left(S^{m}\right)^{T} S^{m}=\left(K^{m}\right)^{T} K^{m},
$$

which implies that inner products between corresponding pairs of columns are all equal and the two matrices have the same coherence. We show that $S^{m}$ is also a sparse matrix.

Proposition 1 Let the rows of $S^{m}$ be indexed by $\left(\begin{array}{ll}u_{1} & u_{2}\end{array}\right)$ where $u_{1}, u_{2} \in \mathbb{Z}_{2}^{m}$ and let the columns of $S^{m}$ be indexed by $\left(\begin{array}{lll}a_{1} & a_{2} & b\end{array}\right)$ where $a_{1}, a_{2}, b \in$ $\mathbb{Z}_{2}^{m}$. Then

$S_{\left(u_{1}, u_{2}\right),\left(a_{1}, a_{2}, b\right)}^{m}= \begin{cases}\frac{1}{2^{m / 2}(-1)^{u_{1} a_{1}^{T}}} & u_{1} P_{b}+u_{2}=a_{2} \\ 0 & \text { otherwise. }\end{cases}$

Proposition 2 Each row of $S^{m}$ has $2^{2 m}$ nonzeros and each column of $S^{m}$ has $2^{m}$ nonzeros.

We will refer to $S^{m}$ as a sparse Kerdock matrix. Figure 1 gives an example of the Sparse Kerdock Frame for the case $m=2(16 \times 64)$.

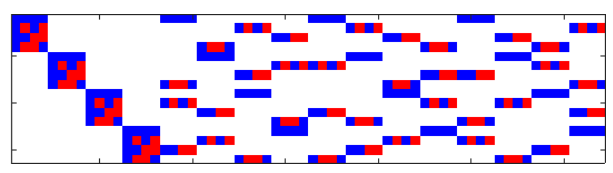

(a) $m=2$

Fig. 1: Example of the Sparse Kerdock Frame $S^{m}$ for $m=2$. Blue + ; red - ; white 0 . 


\section{COMPARISON WITH STEINER EQUIANGULAR TIGHT FRAMES}

In this section we discuss the relative benefits of Sparse Kerdock Frames and the only known infinite families of sparse ETFs: Steiner ETFs. We refer the reader to [12, Table 1] for details on the specifications of the various Steiner ETFs.

Suppose we are interested in infinite families of ETFs which have a similar sparsity and number of frame elements to Sparse Kerdock Frames. An $n \times N$ Sparse Kerdock Frames has column sparsity $k \sim 1 / \sqrt{n}$. The only Steiner ETFs to have sparsity $k \sim 1 / \sqrt{n}$ are those of affine, projective, unitals or Denniston type. Meanwhile Sparse Kerdock Frames have a number of frame elements $N \sim n^{3 / 2}$. The only Steiner ETFs to have $N \sim n^{3 / 2}$ are those of affine and projective type $^{1}$. We see then that the natural competitors are the affine and projective families, since they are the only two families which do not have either significantly fewer columns or significantly greater sparsity.

Advantages of Sparse Kerdock Frames over Steiner ETFs of affine and projective type become apparent by considering the size of the unitary unimodular (complex Hadamard) matrix required for their construction. In the sparse Kerdock case, the requisite size is $2^{m}$, so the frame can have real entries (as already established in Section 2). Furthermore, the fast Walsh-Hadamard Transform (WHT) can be used to compute corresponding mat-vecs with complexity $\mathcal{O}(N \log N)$. This is immediately apparent because a sparse Kerdock matrix can be transformed using the orthogonal matrix $H_{2^{m}} \otimes I_{2^{m}}$ into a DG frame, and it is known that mat-vecs for both DG frames and the orthogonal transformation can be performed in $\mathcal{O}(N \log N)$. Meanwhile Steiner ETFs of affine and projective type require a unimodular unitary matrix of size $q+2$, where $q$ is a prime power. A real Hadamard matrix of this size can never exist, and so affine and projective Steiner ETFs with a maximum number of columns cannot be real. Moreover, $q+2$ cannot itself be a prime

\footnotetext{
${ }^{1}$ Steiner ETFs of Denniston type have at best a slightly inferior number of frame elements $N \sim \frac{1}{2} n^{3 / 2}$.
}

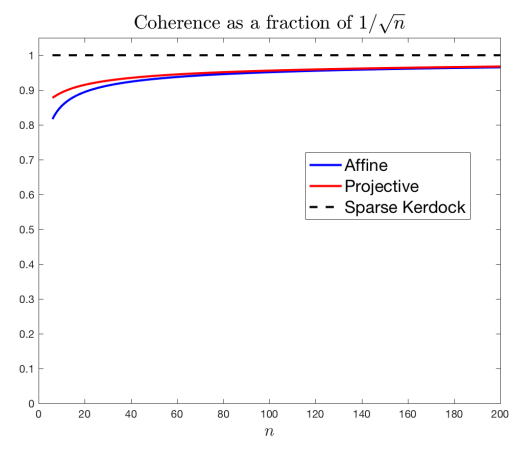

Fig. 2: A comparison of the coherence of Steiner ETFs and Sparse Kerdock Frames for varying $n$.

power, and so the standard fast transforms based upon divide-and-conquer principles are not applicable. It follows that the complexity of computing a mat-vec is determined by the sparsity, leading to an inferior complexity of $\mathcal{O}\left(N^{4 / 3}\right)$.

We note that, unlike Steiner ETFs, Sparse Kerdock Frames are only near-equiangular, and the advantageous properties given above must therefore be traded off against a loss of packing optimality. However, we emphasize that, if $N$ grows superlinearly in $n$,

$$
\sqrt{\frac{N-n}{n(N-1)}} \rightarrow \sqrt{\frac{1}{n}}
$$

as $n \rightarrow \infty$, so the disparity becomes asymptotically small. This effect is illustrated in Figure 2 in which the coherence of optimally-sparse Steiner ETFs of affine and projective type are shown for varying $n$ (as a factor of $1 / \sqrt{n}$ ).

\section{CONCLUSION}

We have proposed a new construction of sparse near-equiangular tight frames which have application in full-duplex communication in ad-hoc wireless networks. We have also highlighted their advantages over Steiner ETFs with similar numbers of columns and sparsity: they are always real, and a fast transform exists for computing their associated matrix-vector products. 


\section{REFERENCES}

[1] W. Alltop. Complex sequences with low periodic correlations. IEEE Transactions on Information Theory, 26(3):350-354, 1980.

[2] L. Applebaum, W. Bajwa, R. Calderbank, and S. Howard. Choir codes: Coding for full duplex interference management. In Allerton Conference on Communication, Control, and Computing, 2011.

[3] L. Applebaum, W. Bajwa, M. Duarte, and R. Calderbank. Multiuser detection in asynchronous on-off random access channels using Lasso. In Allerton Conference on Communication, Control, and Computing, 2010.

[4] L. Applebaum, W. Bajwa, M. Duarte, and R. Calderbank. Asynchronous codedivision random access using convex optimization. Physical Communication, 5(2):129147, 2012.

[5] L. Applebaum, S. Howard, S. Searle, and R. Calderbank. Chirp sensing codes: Deterministic compressed sensing measurements for fast recovery. Applied and Computational Harmonic Analysis, 26(2):283-290, 2009.

[6] B. Bodmann and V. Paulsen. Frames, graphs and erasures. Linear algebra and its applications, 404:118-146, 2005.

[7] R. Calderbank, P. Casazza, A. Heinecke, G. Kutyniok, and A. Pezeshki. Sparse fusion frames: Existence and construction. Advances in Computational Mathematics, 35(1):1-31, 2011.

[8] R. Calderbank, S. Howard, and S. Jafarpour. Construction of a large class of deterministic sensing matrices that satisfy a statistical isometry property. IEEE Journal of Selected Topics on Signal Processing, 4(2), 2010.

[9] P. Casazza, A Heinecke, F. Krahmer, and G. Kutyniok. Optimally sparse frames. IEEE Transactions on Information Theory, 57(11):7279-7287, 2011.
[10] J. Conway, R. Hardin, and N. Sloane. Packing lines, planes, etc.: packing in Grassmannian spaces. Experimental Mathematics, 5(2):139159, 1996.

[11] R. DeVore. Deterministic constructions of compressed sensing matrices. Journal of Complexity, 23:918-925, 2007.

[12] M. Fickus, D. Mixon, and J. Tremain. Steiner equiangular tight frames. Linear algebra and its applications, 436(5):1014-1027, 2012.

[13] D. Guo and L. Zhang. Virtual full-duplex wireless communication via rapid on-offdivision duplex. In Allerton Conference on Communication, Control, and Computing, 2010.

[14] R. Holmes and V. Paulsen. Optimal frames for erasures. Linear Algenra and its Application, 377:31-51, 2004.

[15] J. Jasper, D. Mixon, and M. Fickus. Kirkman equiangular tight frames and codes. IEEE Transactions on Information Theory, 60(1):170-181, 2014.

[16] A. Kerdock. A class of low-rate non-linear binary codes. Information and Control, 20:182187, 1972.

[17] T. Strohmer and R. Heath Jr. Grassmannian frames with applications to coding and communication. Applied and Computational Harmonic Analysis, 14(3):257-275, 2003.

[18] M. Sustik, J. Tropp, I. Dhillon, and R. Heath Jr. On the existence of equiangular tight frames. Linear Algebra and its Applications, 426:619-635, 2007.

[19] L. Welch. Lower bounds on the maximum cross correlation of signals. IEEE Transactions on Information Theory, 20(3):397-399, 1974. 